\title{
Variables de interés clínico en el tratamiento cognitivo-conductual de la adicción a la cocaína: Especificidad de los trastornos de la personalidad
}

\author{
José M. Martínez-González ${ }^{1}$, Natalia Albein-Urios ${ }^{2}$, Oscar Lozano-Rojas³ y Antonio Verdejo-García ${ }^{2,4}$ \\ ${ }^{1}$ Centro Provincial de Drogodependencias de Granada, España \\ ${ }^{2}$ Universidad de Granada, Granada, España \\ ${ }^{3}$ Universidad de Huelva, Huelva, España \\ ${ }^{4}$ Universidad de Monash, Melbourne, Australia
}

\begin{abstract}
Resumen: El objetivo de este estudio fue conocer la relevancia y relación de variables de interacción social con el riesgo de abandono prematuro del tratamiento, el nivel de ajuste psicológico y la calidad de vida, analizando las diferencias que puedan deberse a la presencia de trastornos de la personalidad. La muestra estuvo compuesta por 95 personas que iniciaron el tratamiento por su adicción a la cocaína. El 53.7 \% de los pacientes presentaban trastornos de la personalidad. Los resultados evidencian la importancia de prestar suficiente atención en fases iniciales del tratamiento al estilo de afrontamiento de los problemas, particularmente al centrado en la emoción porque se relaciona con menores niveles de autoeficacia, calidad de vida autopercibida, menor nivel de ajuste psicológico y mayor riesgo de abandono del tratamiento. Estas deficiencias se observan significativamente más en pacientes con trastornos de la personalidad, entre los que se aprecian además menores niveles de autoeficacia, autoestima, optimismo, autocontrol y habilidades sociales.
\end{abstract}

Palabras clave: Variables de interacción psicosocial; afrontamiento; tratamiento; trastornos de la personalidad; cocaína.

\section{Variables of clinical interest in the cognitive behavioral treatment of cocaine addiction: The specificity of personality disorders}

\begin{abstract}
The aim of this study was to examine the association between social interaction variables and premature cessation of treatment, psychological adjustment and quality of life, as a function of co-existing diagnoses of personality disorders. The sample was composed of 95 individuals who commenced treatment for cocaine addiction. Of the sample, 53.7\% had personality disorders diagnoses. The results highlight the relevance of addressing dysfunctional coping styles during early stages of treatment, especially coping styles linked to emotion processing as they associate with lower levels of self-efficacy, lower perceived quality of life, poorer psychosocial adjustment and higher risk of treatment dropout. These deficits are significantly more prominent in individuals with personality disorders, who present lower levels of self-efficacy, self-esteem, optimism, self-control and social skills.
\end{abstract}

Palabras clave: Variables of psycho-social interaction; coping; treatment; personality disorders; cocaine.

El interés en determinadas variables clínicas en el tratamiento de las drogodependencias se explica por su impacto en la evolución de los problemas relacionados con el consumo de drogas y del propio proceso de recuperación. Se conocen algunas, como las habilidades sociales, las expectativas de autoeficacia, la autoestima,
Recibido: 15 octubre 2014; aceptado: 11 febrero 2015.

Correspondencia: José Miguel Martínez González, Centro Provincial de Drogodependencias de Granada, Hospital de San Juan de Dios, San Juan de Dios 11, 18001 Granada, España. Correo-e: jmmgonz@dipgra.es Reconocimientos: Este estudio ha sido financiado por el proyecto COPERNICO del Plan Nacional sobre Drogas (Convocatoria 2009) y la
Red de Trastornos Adictivos (Convocatoria RETICS 2012, Instituto de Salud Carlos III), Grupo Universidad de Granada. Agradecemos a Miguel Angel Ruiz, Ma Sánchez y Manuela Rivero, psicólogos del Centro Provincial de Drogodependencias de Granada, su colaboración en esta investigación. Conflicto de intereses. Los autores declaran que no existe ningún conflicto de intereses. 
etc. (Becoña, Cortés, Arias, et al., 2011) que parecen tener una relevancia significativa y que contribuyen a explicar las causas por las que algunos pacientes tienen más dificultad para dejar de consumir en comparación a otros. Por esta razón, se asumen que determinadas variables deben ser el centro de atención de los programas de intervención, en la medida en que se vinculan a ellas la progresión hacia objetivos terapéuticos facilitadores de la abstinencia a largo plazo. Las expectativas de autoeficacia ocupan un lugar central en la evolución y superación de las drogodependencias. Se asocian a la capacidad de la personas para afrontar las dificultades inherentes a la adicción y los propios retos que entraña un proceso terapéutico (Llorente e Iraurgi, 2008; Sánchez-Hervas, Tomás y Morales, 2004). La autoeficacia parece ser por tanto esencial en la motivación al cambio (Bandura, 1977) y en el propio resultado del tratamiento de las drogodependencias (Condiotte y Lichtenstein, 1981; Solomon y Annis, 1990; Burling, Riley, Molteen y Ziff, 1989; Sánchez-Hervas y Llorente, 2012). En particular, se han asociado con el éxito del tratamiento del alcoholismo (Long, Williams, Midley y Hollin, 2000).

También se ha encontrado que las bajas expectativas de autoeficacia influyen en el craving (DiClemente, Faishurst y Piotrowski, 1995). En particular se observa un craving menos intenso en la medida en que aumenta la autoeficacia (Martínez-González y Verdejo, 2012). Así, intervenciones que potencian las expectativas positivas de autoeficacia se asocian con resultados satisfactorios en el tratamiento (Monras, Mondon y Jon, 2010), mientras que niveles de autoeficacia más bajos incrementan la probabilidad de recaída en el consumo tanto de alcohol como de otras drogas (Walton, Blow, Bingham y Chermack, 2003).

Los estilos de afrontamiento ocupan igualmente un espacio destacado en el tratamiento de las adicciones. El programa de prevención de recaídas de Marlat y Gordon (1985), el programa para el tratamiento de la adicción a la cocaína de Carroll (1998), el programa cognitivo para el tratamiento de las adicciones de Beck (1999) o el programa de reforzamiento comunitario más incentivos para el tratamiento de la adicción a la cocaína (Budney y Higgins, 1998) confían parte de su desarrollo al entrenamiento de habilidades cognitivo-conductuales de afrontamiento de situaciones de riesgo. En especial se sabe que el estilo de afrontamiento de estados emocionales negativos es uno de los retos diferenciales del tratamiento de las drogodependencias cuando los pacientes presentan a su vez un trastorno de la personalidad (TP) (Martínez-González y Trujillo, 2003). Habitualmente los programas de tratamiento de corte cognitivo-conductual incluyen entre sus componentes el entrenamiento en habilidades de afrontamiento de diversa índole por considerarlos parte esencial del proceso de cambio de los hábitos de consumo de la persona y del nivel de autocontrol del paciente (Kadden, 1999; Secades-Villa, García-Rodríguez, Fernández y Carballo, 2007). Sin duda estas variables actúan bajo un modelo complejo en el que cada una de ellas influyen interconectadas entre sí y a lo largo de todo el tratamiento, como sucede por ejemplo con la autoeficacia y el estilo de afrontamiento donde se ha visto que niveles elevados de autoeficacia se correlaciona con estrategias de afrontamiento más eficaces (Marlatt y Gordon, 1985).

El objetivo de este trabajo, que forma parte de una investigación más amplia (Albein-Urios et al., 2013; Martínez-González, Albein-Urios, Lozano y Verdejo-García, 2014), fue analizar la relación de variables de interacción social como la autoeficacia, autoestima, optimismo, locus de control, habilidades sociales y de afrontamiento; con indicadores de riesgo de abandono, el ajuste psicológico o la presencia de TP, en la medida en que la conexión entre estas variables puede aportar información muy útil para el diseño de intervenciones más efectivas. Creemos que puntuaciones en variables esenciales del tratamiento van a ser significativamente diferentes entre los paciente en función de la presencia de TP.

\section{Método}

\section{Participantes}

La muestra la componen 95 pacientes con trastorno por dependencia a la cocaína, que inician tratamiento en el Centro Provincial de Drogodependencias de Granada. La media de edad de los participante fue de 32.6 años en un rango de edad de 19 a 52 años. El $87.4 \%$ eran hombres $(n=83)$ mientras que el $12.6 \%$ mujeres $(n=12)$. La evaluación de la dependencia de sustancias y psicopatología comórbida la llevaron a cabo psicólogos clínicos del centro durante el primer mes de tratamiento, atendiendo a los criterios que permiten realizar un correcto diagnóstico dual. El $43.71 \%$ de los pacientes presentaban psicopatología no psicótica del Eje I (DSM-IV-TR, 2002) en el momento de la evaluación. Presentaban un TP el $53.7 \%(n=51)$ de la muestra, siendo la distribución de TP la siguiente: antisocial $6.3 \%(n=6)$, límite $16.8 \%(n=16)$, histriónico $11.6 \%(n=11)$, narcisista $1.1 \%(n=1)$, evitativo $11.6 \%(n=11)$, obsesivo-compulsivo $6.3 \%(n=6)$. Quedaron excluidos del estudio los pacientes con psicopatología psicótica, trastornos del estado de ánimo graves o que no pudiesen comprender y contestar a los cuestionarios. A todos los pacientes se les solicitó el consentimiento informado. 


\section{Instrumentos}

Cuestionario de Variables de Interacción Psicosocial (VIP; Pedrero, Pérez, De Ena y Garrido, 2005). El cuestionario lo componen 84 ítems que se agrupan en nueve escalas: autoestima, autoeficacia, optimismo, locus de control, habilidades sociales, autocontrol, afrontamiento centrado en la tarea, afrontamiento centrado en la emoción y afrontamiento centrado en la búsqueda de apoyo. Utiliza respuestas tipo Likert de cuatro niveles: muy de acuerdo, de acuerdo, en desacuerdo y muy en desacuerdo. Puntuaciones más altas implican más presencia de la dimensión, si bien es cierto que en el caso del afrontamiento centrado en la emoción lo deseable son puntuaciones negativas ya que representan una menor tendencia de la persona a utilizar esta estrategia. En el caso de la escala de locus de control, las puntuaciones más elevadas corresponden a un locus de control interno mientras que puntuaciones más bajas corresponden a un locus de control externo. La fiabilidad del cuestionario analizada a través de la consistencia temporal es de .69, siendo la correlación entre escalas de .80 .

Cuestionario General sobre Salud - General Health Questionnarie (GHQ-28; Goldber y Hiller, 1979). Evalúa psicopatología no psicótica. Establece un índice de gravedad de ajuste psicológico construido a partir de la suma de cuatro subescalas: síntomas somáticos, ansiedad, disfunción social y depresión (González-Saiz, et al., 1997). La consistencia interna del cuestionario es de 0.93 .

Examen Internacional de los Trastornos de la Personalidad (IPDE; Loranger, et al. 1991; versión española de López-Ibor, Pérez Urdániz y Rubio, 1996). Entrevista semiestructurada para la evaluación y diagnóstico de los TP. Este instrumento permite discriminar más fácilmente la sintomatología controlando satisfactoriamente la contaminación entre trastornos clínicos y trastornos de la personalidad (DSM).

Structured Clinical Interview for DSM IV Axis I (SCID-I; First, Spitzer, Gibbon y Williams, 1999). Evalúa la presencia de psicopatología del Eje I atendiendo a criterios DSM-IV-TR (APA, 2002). Permite identificar los casos de dependencia de sustancias.

Personality Beliefs Questionnaire (PBQ; Beck y Beck, 1991). Proporciona diferentes perfiles de personalidad. Es un cuestionario diseñado para medir el grado con el que una persona se identifica con creencias básicas disfuncionales relacionadas con los TP. Consta de 126 ítems, incluyendo diez escalas correspondientes a cada uno de los trastornos del Eje II (DSM-IV-TR, 2002). Nueve de las escalas están compuestas por 14 ítems. Las escalas son: evitativo, dependiente, obsesivo-compulsivo, narcisista, antisocial, histriónico, esquizoide y paranoide. Una escala adicional mide el trastorno límite de la personalidad, compuesta de 14 ítems extraídos del resto de escalas. Presenta respuestas tipo likert de cinco valores. Los coeficientes alfa de las escalas de personalidad oscilan desde .64 a .89 .

Cuestionario de Predicción del Abandono (VPA-30; Sirvent, 2010). Evalúa el nivel de riesgo de abandono del tratamiento. Consta de 30 ítems con una escala general de 14 ítems y 5 subescalas o factores que recogen variables de adherencia terapéutica: motivación, conciencia de problema, sentimientos adversos, tratamiento $\mathrm{y}$ adherencia. A mayor puntuación en cada una de las dimensiones corresponde un mayor riesgo de abandono del tratamiento durante los dos siguientes meses. La consistencia interna del cuestionario es de .74.

Test para la Evaluación de la Calidad de Vida en Adictos a Sustancias Psicoactivas (TECVASP; Lozano et al., 2006). Es una medida específica de la calidad de vida (CV) en drogodependientes. Consta de 20 ítems con formato de respuesta graduada con cinco categorías: nada, poco, a veces, bastante, mucho. Puntuaciones altas en el test indican buena CV relacionada con la salud y puntuaciones bajas es un indicador de una mala CV. Los valores de consistencia interna en diferentes estudios oscilan de .79 a .84 .

\section{Procedimiento}

La caracterización psicopatológica de la muestra la realizaron psicólogos del Centro Provincial de Drogodependencias de Granada durante las sesiones de evaluación y los cuestionarios VIP, VPA-30, GHQ-28 y PBQ los completaron los pacientes en sus hogares, entregándolos tras una semana desde que se realizó la evaluación inicial. Se realizaron dos evaluaciones, la primera antes de que finalizara el primer mes de tratamiento y en condiciones de abstinencia que permitieron realizar el diagnóstico psicopatológico comórbido con garantías. La segunda evaluación se llevó a cabo a los tres meses del inicio del tratamiento.

\section{Tratamiento estadístico de los datos}

Se utilizaron estadísticos descriptivos para caracterizar la muestra. Las comparaciones entre grupos e intrasujetos se realizaron aplicando $t$ de Student y ANOVA, según procede por el tipo de variable. Se analizaron diferentes correlaciones bivariadas y se aplicó un análisis de regresión lineal para conocer la capacidad explicativa de algunas variables como la autoeficacia o habilidades de afrontamiento sobre el riesgo de abandono del trata- 
miento. Los análisis estadísticos fueron realizados con el programa SPSS .19.0.

\section{Resultados}

El análisis de la relación entre las dimensiones del cuestionario VIP muestra que una mayor autoeficacia se relaciona de forma negativa y estadísticamente significativa con el afrontamiento centrado en las emociones $(r=$ $0.281 ; p<0.05)$ y positiva con el estilo de afrontamiento centrado en la tarea $(r=0.654 ; p<0.001)$.

\section{Calidad de vida y variables de interacción social}

En la tabla 1 se observa que la calidad de vida autopercibida más alta se asocia significativamente a mayores niveles en autocontrol, habilidades de afrontamiento centradas en la terea, locus de control interno, autoeficacia y autoestima. En el caso de las estrategias de afrontamiento centradas en la emoción, la relación con la calidad de vida es negativa, lo que muestra que en la medida en que mejora la calidad de vida de los pacientes tienden a utilizar menos estrategias centradas en el afrontamiento emocional.

\section{Ajuste psicológico}

En lo que se refiere a la relación de las variables del cuestionario VIP con la escala general del cuestionario GHQ-28, se aprecia que mayor nivel de gravedad de ajuste psicológico se relaciona significativamente e in- versamente con el autocontrol, habilidades de afrontamiento en la tarea, las habilidades sociales, el locus de control interno, el optimismo, la autoeficacia y la autoestima. En sentido positivo se relaciona con las habilidades centradas en el afrontamiento emocional lo que muestra que mayores niveles de malestar propician la tendencia a presentar respuestas de afrontamiento centradas en la eliminación de las emociones negativas (Tabla 1). En la tabla pueden consultarse las relaciones específicas de cada variable con las diferentes escalas del cuestionario de gravedad de ajuste psicológico.

\section{Variables de interacción social en función de la personalidad.}

El análisis comparativo de los factores del cuestionario VIP en función de la presencia de TP muestra que existen diferencias estadísticamente significativas en seis de ellos. Puede comprobarse que en lo que se refiere a las habilidades de afrontamiento solo hay diferencias en el afrontamiento emocional, siendo significativamente mayor la tendencia a utilizar esta estrategia en el grupo de pacientes con TP. Con respecto al autocontrol, habilidades sociales, optimismo, autoeficacia y autoestima las puntuaciones son significativamente más elevadas en el grupo de pacientes que no presenta un TP (Tabla 2.).

El análisis detallado de los esquemas prototípicos de cada TP en relación con el sistema atribucional de los pacientes muestra que las puntuaciones del cuestionario PBQ se correlaciona de forma positiva y estadísticamente significativa con el locus de control, lo que muestra

Tabla 1. Correlaciones entre las dimensiones del Cuestionario de Variables de Interacción Psicosocial (VIP), el ajuste psicológico (GHQ-28) y la calidad de vida (TECVASP)

\begin{tabular}{|c|c|c|c|c|c|c|}
\hline Dimensiones del cuestionario VIP & $\begin{array}{l}\text { GHQ28A } \\
\text { Síntomas } \\
\text { somáticos }\end{array}$ & $\begin{array}{l}\text { GHQ28B } \\
\text { Ansiedad }\end{array}$ & $\begin{array}{c}\text { GHQ28 } \\
\text { C } \\
\text { Disfunción } \\
\text { social }\end{array}$ & $\begin{array}{c}\text { GHQ28 } \\
\text { D } \\
\text { Depresión }\end{array}$ & $\begin{array}{l}\text { GHQ28 } \\
\text { total }\end{array}$ & $\begin{array}{l}\text { Puntuación } \\
\text { total } \\
\text { TECVASP }\end{array}$ \\
\hline Autocontrol & $-0.362^{* *}$ & $-0.292^{*}$ & $-0.332^{* *}$ & $-0.282^{*}$ & $-0.367^{* *}$ & $0.488^{* *}$ \\
\hline $\begin{array}{l}\text { Habilidades de afrontamiento centradas en el apo- } \\
\text { yo social. }\end{array}$ & 0.015 & -0.027 & 0.095 & -0.025 & 0.018 & 0.094 \\
\hline $\begin{array}{l}\text { Habilidades de afrontamiento centradas en las } \\
\text { emociones. }\end{array}$ & 0.218 & $0.242^{*}$ & $0.318^{* *}$ & $0.349^{* *}$ & $0.334^{* *}$ & $-0.350^{* *}$ \\
\hline Habilidades de afrontamiento centradas en la tarea. & $-0.363^{* *}$ & $-0.283^{*}$ & $-0.431^{* *}$ & $-0.340^{* *}$ & $-0.407^{* *}$ & $0.268^{*}$ \\
\hline Habilidades sociales. & -0.228 & -0.202 & $-0.272^{*}$ & $-0.318^{* *}$ & $-0.304^{*}$ & 0.140 \\
\hline Locus de control. & $-0.349^{* *}$ & $-0.263^{*}$ & $-0.268^{*}$ & $-0.358^{* *}$ & $-0.362^{* *}$ & $0.294^{*}$ \\
\hline Optimismo & -0.225 & -0.188 & $-0.365^{* *}$ & $-0.489^{* *}$ & $-0.376^{* *}$ & 0.211 \\
\hline Autoeficacia & $-0.350^{* *}$ & $-0.273^{*}$ & $-0.378^{* *}$ & $-0.467^{* *}$ & $-0.434^{* *}$ & $0.281^{*}$ \\
\hline Autoestima & $-0.356^{* *}$ & $-0.328^{* *}$ & $-0.426^{* *}$ & $-0.545^{* *}$ & $-0.489^{* *}$ & $0.348^{* *}$ \\
\hline
\end{tabular}

Nota. El $n$ es de 68 excepto para las correlaciones con la puntuación total del cuestionario TECVASP que es de 70. VIP = Cuestionario de Variables de Interacción Psicosocial; GHQ-28 = General Health Questionnarie; TECVASP = Test para la Evaluación de la Calidad de Vida en Adictos a Sustancias Psicoactivas. $* p<.05 ; * * p<.01$. 
Tabla 2. Comparaciones estadísticamente significativas de las puntuaciones del Cuestionario de Variables de Interacción Psicosocial (VIP) en función de la presencia de trastornos de la personalidad

\begin{tabular}{|c|c|c|c|c|c|c|}
\hline $\begin{array}{l}\text { Dimensiones del } \\
\text { cuestionario VIP }\end{array}$ & Grupos & Media & $D T$ & $t$ & $p$ & $d$ \\
\hline \multirow[t]{2}{*}{ Autocontrol } & Presenta trastorno de la personalidad. & 3.37 & 30.07 & 2.490 & 0.015 & -0.5957 \\
\hline & No presenta trastorno de la personalidad. & 21.57 & 31 & & & \\
\hline \multirow[t]{2}{*}{ Afrontamiento emocional. } & Presenta trastorno de la personalidad. & 0.64 & 33.82 & 2.207 & 0.031 & 0.519 \\
\hline & No presenta trastorno de la personalidad. & -17.51 & 34.97 & & & \\
\hline \multirow[t]{2}{*}{ Habilidades sociales } & Presenta trastorno de la personalidad. & 16.97 & 46.00 & -2.943 & 0.004 & -0.7073 \\
\hline & No presenta trastorno de la personalidad. & 44.27 & 28.42 & & & \\
\hline \multirow[t]{2}{*}{ Optimismo. } & Presenta trastorno de la personalidad. & 8.40 & 42.46 & -2.611 & 0.011 & -0.6345 \\
\hline & No presenta trastorno de la personalidad. & 30.42 & 24.62 & & & \\
\hline \multirow[t]{2}{*}{ Autoeficacia. } & Presenta trastorno de la personalidad. & 25.10 & 37.27 & -1.997 & 0.049 & -0.4773 \\
\hline & No presenta trastorno de la personalidad. & 40.87 & 28.19 & & & \\
\hline \multirow[t]{2}{*}{ Autoestima. } & Presenta trastorno de la personalidad. & 9.32 & 49.24 & -3.940 & 0.000 & -0.9561 \\
\hline & No presenta trastorno de la personalidad. & 48.30 & 30 & & & \\
\hline
\end{tabular}

Nota. VIP: Cuestionario de Variables de Interacción Psicosocial.

Tabla 3. Correlaciones de Pearson entre la escala locus de control (VIP) y los tipos de trastornos de personalidad (PBQ) $(N=61)$

\begin{tabular}{ccccccccc}
\hline & TPE & TPD & TAP & TO-CP & TNP & THP & TPP & TLP \\
\hline VIP Locus de control & $-0.310^{*}$ & $-0.454^{* *}$ & $-0.402^{* *}$ & $-0.264^{*}$ & $-0.392^{* *}$ & $-0.263^{*}$ & $-0.373^{* *}$ & $-0.418^{* *}$ \\
\hline
\end{tabular}

Nota. VIP: Cuestionario de Variables de Interacción Psicosocial; PBQ: Personality Beliefs Questionnaire;TPE: trastorno de la personalidad por evitación; TPD: trastorno de la personalidad por dependencia; TAP: trastorno antisocial de la personalidad; TO-CP: trastorno obsesivo-compulsivo de la personalidad; TNP: Trastorno narcisista de la personalidad; THP: trastorno histriónico de la personalidad; TPP: trastorno paranoide de la personalidad: TLP: trastorno límite de la personalidad. $* \mathrm{p}<.05 ; * * \mathrm{p}<.01$.

que los pacientes presentan un sistema atribucional externo en la medida en que se identifican más con estas creencias (Tabla 3 ).

\section{Riesgo de abandono.}

Con el objetivo de conocer la relación entre algunas variables se realizaron diversos análisis de regresión lineal. Mostraron que dimensiones como la habilidades de afrontamiento centrado en la terea y la centrada en las emociones predicen, aunque en sentido diferente, el nivel de riesgo de abandono del tratamiento medido con el cuestionario VPA-30. El afrontamiento centrado en la tarea predice en sentido negativo el riesgo de abandono, indicando que en la medida en que los pacientes tienden a utilizar este estilo de afrontamiento disminuye significativamente el riesgo de abandono del tratamiento. Por el contrario, en la medida en que el afrontamiento se produce centrado en las emociones, mayor es el riesgo de abandono durante los dos primeros meses de tratamiento. El riesgo de abandono también se relaciona con las habilidades de autocontrol y un locus de control interno más robusto porque en la medida en que aumentan éstas dismi- nuye el riesgo de abandono. Por otro lado, el nivel de autoeficacia predice en sentido negativo el nivel general de gravedad de ajuste psicológico, esto es, en la medida en que aumenta la autoeficacia se espera una disminución significativa del malestar psicológico (Tabla 4).

Además, el análisis de las correlaciones entre dimensiones del cuestionario VIP y los índices del cuestionario VPA-30 refleja que tanto la autoeficacia como la autoestima se relacionan de forma significativa con distintos dimensiones del cuestionario. La autoeficacia se relaciona significativamente y de forma negativa con la escala "sentimientos adversos" del cuestionario VPA-30 $(r=-0.622 ; p=0.037)$ a la vez que la autoestima se relaciona también de forma negativa con la dimensión "motivación al tratamiento" $(r=-0.757 ; p=0.011) \mathrm{y}$ positiva con la "percepción de utilidad del tratamiento" $(r=0.717 ; p=0.020)$.

\section{Análisis de la evolución de las estrategias de afrontamiento.}

La comparación de las puntuaciones del cuestionario VIP al inicio del tratamiento y a los tres meses $(n=21)$ 
Tabla 4. Análisis de regresión lineal, siendo variables predictoras las escalas del VIP y variables criterio el riesgo de abandono (VPA-30) y el ajuste psicológico (GHQ-28)

\begin{tabular}{lll}
\hline \multicolumn{1}{c}{ Variable predictora } & \multicolumn{1}{c}{ Variable criterio } \\
\hline Habilidades centradas en la terea. & Riesgo de abandono & $\left(\beta=-0.626 ; p=0.002 ; R^{2}=.39\right)$ \\
Habilidades centradas en las emociones. & Riesgo de abandono & $\left(\beta=0.004 ; p=0.027 ; R^{2}=0.23\right)$ \\
Habilidades de autocontrol. & Riesgo de abandono & $\left(\beta=-0.461 ; p=0.035 ; R^{2}=0.21\right)$ \\
Locus de control & Riesgo de abandono & $\left(\beta=-0.002 ; p=0.588 ; R^{2}=.016\right)$ \\
Autoeficacia & Ajuste psicológico & $\left(\beta=-0.450 ; p=0.005 ; R^{2}=0.20\right)$ \\
\hline
\end{tabular}

Nota: VIP = Cuestionario de Variables de Interacción Psicosocial; VAP-30: Inventario de Autoengaño y Mixtificación; GHQ-28 = General Health Questionnarie

nos muestra que a los tres meses de tratamiento $(M=$ -22.61, $D T=35.96$ ) los pacientes utilizan significativamente menos estrategias de afrontamiento centradas en las emociones que al inicio del tratamiento $(M=-6.57$, $D T=36.85)[t(19)=2.38, \mathrm{p}<.05]$.

\section{Discusión}

Los resultados del estudio confirman nuestra hipótesis de trabajo en la medida en que la presencia de variables esenciales en el tratamiento difiere en función de los TP. El análisis de la relación entre las variables del cuestionario VIP y la CV autopercibida muestra que en la medida en que el paciente percibe una mejor calidad de vida mejoran significativamente al inicio del tratamiento variables tan relevantes como el autocontrol, las habilidades de afrontamiento centradas en la tarea, el locus de control interno, la autoeficacia y la autoestima. Esto confirma la importancia otorgada por diversos autores a la incorporación en los programa de prevención de recaídas el entrenamiento o mejora de las variables mencionadas (Secades-Villa, et al., 2007). Una mejor CV al iniciar el tratamiento se relaciona con una menor tendencia del individuo a centrarse en la eliminación del malestar en lugar de actuar sobre la causa que le provoca el malestar. Esto es un indicador del hecho de que el paciente cuente con un repertorio conductual más favorable para rentabilizar los primeros pasos del tratamiento. La CV tiene un papel destacado en esos momentos y en este sentido también se destacó en otros trabajos el valor de la satisfacción de la persona como variable relevante en el desarrollo del tratamiento (Larsen, Attkinsson, Hargreaves y Nguyent, 1979; LaBounty, Hatsukami, Morgan y Nelson, 1992; Martínez-González, Graña y Trujillo, 2011).

El vínculo que se observa entre el nivel de ajuste psicológico o malestar general y las dimensiones del cuestionario VIP denotan la necesidad de prestar atención a esta relación. Los resultados indican que el nivel de malestar general al iniciar el tratamiento parece afectar ne- gativamente a variables tan relevantes como el autocontrol, las habilidades de afrontamiento en la tarea, las habilidades sociales, el locus de control, el optimismo, la autoeficacia y la autoestima. Estos resultados contribuyen a poner en valor la importancia concedida a variables como el malestar general entre los componentes esenciales del modelo de prevención de recaídas (Hendershot, Witkiewitz, George y Marlatt, 2011) que destaca el peso que puede llegar a tener porque podría inducir al paciente a emitir respuestas de afrontamiento centradas en la búsqueda de alternativas para evitar el malestar emocional. No obstante se ha podido comprobar que los pacientes parecen utilizar significativamente menos las estrategias de afrontamiento centradas en la emoción a los tres meses de tratamiento en comparación al estilo de afrontamiento que utilizaban al iniciarlo.

El análisis de regresión indica que algunas escalas del cuestionario VIP predicen el riesgo de abandono. Se observa que al inicio del tratamiento aumenta el riesgo en la medida en que los paciente utilizan como estrategia de afrontamiento la búsqueda de la eliminación del malestar emocional, esto es, los pacientes que persiguen la eliminación del malestar en detrimento de otras estrategias de afrontamiento como por ejemplo las centradas en la tarea, presentan mayor riesgo de abandono a lo largo de los dos primeros meses. Hemos podido comprobar en esta investigación que la eliminación del malestar como estrategia fundamental de afrontamiento constituye un factor de riesgo significativo para el abandono prematuro del tratamiento.

Por el contrario se constata que este riesgo disminuye significativamente cuando el paciente utiliza la estrategia de afrontamiento centrada en la tarea, que persigue el análisis y puesta en marcha de tareas que facilitan la resolución de un conflicto o dificultad. Lo mismo sucede con niveles más altos de autocontrol del paciente que se relaciona con una disminución significativa del riesgo de abandono. Esto sugiere que al iniciar el tratamiento se debería prestar atención y potenciar de un modo especial las intervenciones destinadas a mejorar tanto el autocon- 
trol como el empleo de estrategias de afrontamiento centradas en las tareas.

Por otro lado hemos podido comprobar que un sistema atribucional más externo conlleva mayor riesgo de abandono del tratamiento, probablemente porque en estas condiciones el paciente cree que su mejoría o éxito en el tratamiento depende en mayor medida de variables externas y ajenas a él, como la ayuda familiar o tener un trabajo. Por esta razón es deseable que el paciente emplee un sistema atribucional interno que le permita enfrentarse de un modo proactivo a las dificultades esperables en un proceso de recuperación, sin esperar a que factores externos lo liberen del esfuerzo que debe necesariamente hacer para la superación de las dificultades. Los diferentes tipos de TP presentan niveles desiguales entre sí en lo que se refiere al locus de control, destacando la importancia de adecuar las intervenciones de esta psicopatología considerando el estilo atribucional de cada uno de ellos.

La autoeficacia se relaciona significativamente con el riesgo de abandono prematuro del tratamiento, particularmente disminuyendo el riesgo que viene dado por los sentimientos adversos (VPA-30). Esto es, al inicio del tratamiento los pacientes con mejores expectativas de autoeficacia les afectan significativamente menos los sentimientos adversos (Sirvent, 2010; Martínez-González, Albein-Urios, Lozano y Verdejo, 2014), lo que constata que las expectativas de autoeficacia positivas disminuyen las dificultades para enfrentarse a estados emocionales negativos. Estos resultados destacan la enorme importancia que tienen al iniciar el tratamiento las expectativas de autoeficacia porque de una manera significativa se vinculan con la experimentación de malestar (Chicharro, Pedrero y Pérez, 2007). Si la mejora de las expectativas de autoeficacia disminuye la dificultad con la que el paciente afronta determinados síntomas que le provocan malestar, como pudo comprobarse también en el estudio de Rueda y Pérez-García (2004), debería asumirse como objetivo prioritario del tratamiento el aumento de las expectativas de autoeficacia toda vez que de ese modo disminuyen la intensidad de determinados síntomas concernientes tanto al trastorno por dependencia a las sustancias como a los TP (Beck, Wrihgt, Newman y Liese, 1999; Llorente e Iraurgi, 2008).

El análisis de la autoeficacia muestra aspectos que pueden ser muy esclarecedores en la práctica clínica del tratamiento cognitivo de la adicción a la cocaína (Llorente e Iraurgi, 2008). La relación entre autoeficacia y estrategias de afrontamiento apoyan modelos en los que se vincula una mayor autoeficacia con estrategias de afrontamiento más adaptativas (Marlatt y Gordon, 1985). En ésta ocasión hemos constatado que los pacientes con- sumidores de cocaína que presentan mayor nivel de autoeficacia utilizan significativamente menos estrategias centradas fundamentalmente en la eliminación del malestar emocional, porque los pacientes que presentan menores expectativas de autoeficacia tienden a utilizar, al menos al iniciar el tratamiento, estrategias para eliminar su malestar en lugar de dirigirse hacia las causas. En definitiva los pacientes con mejores expectativas de autoeficacia suelen emplear estrategias centradas en la tarea que como se sabe analizan el problema, las opciones más apropiadas y utilizan estrategias oportunas para el afrontamiento de cada problema.

Se constatan diferencias esenciales al iniciar el tratamiento en consumidores de cocaína dependiendo de la presencia de TP. Los que no presentan esta psicopatología presentan mejores puntuaciones en variables tan relevantes como autocontrol, habilidades sociales, optimismo, autoeficacia y autoestima. A diferencias de los pacientes sin este tipo de psicopatología, los que presentan un TP tienden a buscar la pronta eliminación de estados emocionales negativos como estrategia principal de afrontamiento en lugar de actuar sobre las causas del malestar, lo que está en consonancia con la conocida funcionalidad que tienen el consumo de drogas en éstos pacientes (Martínez-González y Trujillo, 2003). En definitiva los resultados evidencian la tendencia de los pacientes con TP a utilizar estrategias de afrontamiento menos adaptativos (Monras, Mondon y Jon, 2010).

En la medida en que los pacientes se identifican más con las creencias nucleares de cada uno de los trastornos de la personalidad se potencia su locus de control externo, lo que es coherente con la clínica de esta psicopatología por que estos pacientes suelen exculparse para atribuir a otros de sus propios errores, hasta el extremo de creer que los problemas que sufren suelen tener un origen externo a ellos mismos (Beck y Freeman, 1995).

Además, comprobamos que los pacientes con TP presentan significativamente menor nivel de expectativas de autoeficacia en comparación al resto. En un estudio anterior pudimos constatar este resultado en pacientes consumidores de alcohol (Martínez-González y Verdejo, 2012).

El análisis más pormenorizado en la muestra de pacientes que presentan un TP indica que las expectativas positivas de autoeficacia predicen en sentido inverso el nivel de malestar general (Chicharro, 2005). El resultado sin lugar a dudas es de gran calado para la intervención en la medida en que estos pacientes pueden afrontar más fácilmente el malestar cuando presentan niveles más elevados de autoeficacia. Así, y de un modo más específico puede decirse que se convierte también para los pacien- 
tes con TP en una variable extraordinariamente significativa en el transcurso del tratamiento (Beck y Freeman, 1995; Urbanoski, Kelly, Hoeppner y Slayma, 2012), fundamentalmente porque las expectativas de autoeficacia reducen las complicaciones (Van Beck y Verheul, 2008). En este sentido, cuando los pacientes con TP confían más en sus propias capacidades presentan menos problemas para sortear las dificultades propias del afrontamiento de un índice de malestar psicológico mayor, lo que disminuye también de forma específica entre estos pacientes.

De acuerdo con los resultados del estudio sobre el riesgo de abandono en pacientes con TP en que se mostraba que la psicopatología por sí sola no está asociada al abandono del tratamiento (Martínez-González, Albein-Urios, Lozano y Verdejo, 2014), puede decirse que el riesgo de abandono viene dado por variables como el estilo de afrontamiento de problemas, el autocontrol, autoeficacia o el sistema atribucional, convirtiéndose estas por tanto en un aspecto central en las primeras etapas del tratamiento.

Este trabajo pone de relieve la necesidad de potenciar y priorizar intervenciones terapéuticas centradas en variables como el autocontrol, estilos de afrontamiento, habilidades sociales, locus de control interno, autoeficacia y autoestima, porque no solamente mejorará el nivel de gravedad de ajuste y afrontamiento de determinados síntomas de gran calado en la evolución sino que disminuye el riesgo de abandono del tratamiento durante los dos primeros meses de tratamiento, especialmente cuando se trata de pacientes con TP.

Creemos que este trabajo podría enriquecerse con un análisis de las variables a largo plazo, por lo que una posible limitación guarda relación con el número de mediciones realizadas. En futuras investigaciones se realizarán evaluaciones de estas variables en un mayor número de momentos temporales del proceso de tratamiento.

\section{Referencias}

APA, (2002). DSM-IV-TR Manual diagnóstico y estadístico de los trastornos mentales, IV Texto Revisado. Barcelona: Masson.

Albein-Urios, N, Martínez-González, J.M., Lozano, O., Moreno, L., Soriano-Mas, C., y Verdejo, A. (2013). Negative urgency, disinhibition and reduce temporal pole gray matter characterize the comorbidity of cocaine dependence and personality disorders. Drug and Alcohol Dependence, 132, 231-7.

Bandura, A. (1977). Self-efficacy: Toward a unifying theory of behavioral change. Psychological Review, 84, 191-295.

Beck, A.T. y Freeman, A. (1995). Terapia cognitiva de los trastornos de personalidad. Barcelona: Paidos.

Beck, A. T., y Beck, J. S. (1991). The Personality Belief Questionnaire. Unpublished assessment instrument. Bala Cynwyd, PA: The Beck Institute for Cognitive Therapy and Research.
Beck, A.T., Wrihgt, F. D., Newman, C.F. y Liese, B.S. (1999). Terapia cognitiva de las drogodependencias. Barcelona: Paidos.

Becoña, E., Cortés, M., Arias, F., Barreiro, C., Berdullas, J. Iraurgui, I., Llorente, J.M, López, A., Madoz, A., Martínez-González, J.M., Ochoa, E., Palau, C., Palomares, A., Villanueva, V. (2011). Manual de Adicciones para Psicólogos Especialistas en Psicología Clínica en Formación. Barcelona: Socidrogalcohol.

Bobes, J., González, G., Portilla, M., Iglesias, C. y Wallace, D. (1995). Adaptación Europea del Addiction Severity Index. Manual para el entrenamiento y administración de la entrevista EuropASI. Universidad de Oviedo.

Budney, A. J. y Higgins, S. T. (1998). A community reinforcement plus vouchers approach: Treating cocaine addiction. Rockvi1le, MD: National Institute on Drug Abuse.

Burling, T. A., Riley, P. M., Molteen, J. O. y Ziff, D. C. (1989). Self-efficacy and relapse among in-patient drug and alcohol abusers: A predictor of outcome. Journal of Studies on Alcohol, 50, 354-360.

Carroll, K. M. (1998). A Cognitive-Behavioral Approach: Treating Cocaine Addiction. Rockville, M.D.: National Institute on Drug Abuse.

Condiotte, M., \& Lichtenstein, E. (1981). Self-efficacy and relapse in smoking cessation programs. Journal of Clinical and Consulting Psychology, 49, 641- 658.

Chicharro, J. (2005). Variables indicadoras del tratamiento y evolución en pacientes drogodependientes. Su relación con rasgos de personalidad. Psicología.com. 9 (2).

Chicharro, J., Pedrero, E., y Pérez, M. (2007). Autoeficacia para resistirse al consumo de sustancias como predictora de resultados del tratamiento y su relación con variables de personalidad: estudio de una muestra de adictos con DICQ, el VIP y el MCMII-II. Adicciones, 19, 141-152.

DiClemente, C. C., Fairhurst, S. K., \& Piotrowski, N. A. (1995). Self-Efficacy and addicitive behaviors. In J. E. Maddux, ed., Self-Efficacy, adaptation, and adjustment: Theory, research, and application (pp. 109-141). New York: Plenum.

First, M., Spitzer, R., Gibbon, M. y Williams, J. (1999). Guía del usuario para la entrevista clínica estructurada para los trastornos de la personalidad del Eje I del DSM-IV (SCID-I). Madrid: Masson.

Golberg, D.P. y Hiller, V.F. (1979). A scaled version of the General Health Questionaire. Windsor: NFER Publishing Company.

González-Saiz, F.M., Carulla, S., Martínez J.M., López, A., Ruiz, J. y Guerra, D. (1997). Indicador del tratamiento de la adicción a opiáceos. Cádiz: Servicio de Publicaciones de la Universidad de Cádiz.

Hendershot, C., Witkiewitz, K., George, W. y Marlatt, A. (2011). Relapse prevention for addictive behaviors. Substance Abuse Treatment, and Policy, 6, 17.

Kadden, R.M. (1999). Cognitive behavior therapy: In P. J. Ott, R. E. Taster and Ammeman. Sourcebook on substance Abuse: Etiology, Epidemiology, Assesment and Treatment (pp. 272283). Boston: Allyn and Bacon.

LaBounty, L. P., Hatsukami, D., Morgan, S. F., \& Nelson, L. (1992). Relapse among alcoholics with phobic and panic symptoms. Addictive Behaviors, 17, 9-15.

Larsen, D. L., Attkinsson, C. C., Hargreaves, W. A., \& Nguyent, D. (1979). Assessment of client/patient satisfaction: Develop- 
ment of a general scale. Evaluation and Programme Planning, 2, 197-207.

Llorente, J.J. e Uraurgi, E.I. (2008). Tratamiento cognitivo conductual aplicado en la deshabituación de cocaína. Trastornos Adictivos, 10, 252-74.

Long, C.G., Williams, M, Hollin, C.R. (1999). Within treatment factors as predictors of drinking outcome following cognitive-behavioral treatment. Addiction Biology, 4 (2), 238-239.

Lozano, O.M., Rojas, A.J., y Pérez, C. (2006). Construcción de un test para medir la calidad de vida relacionada con la salud especifico para drogodependientes. Sevilla: Junta de Andalucía.

Marlatt, G.A. y Gordon, J.R. (Eds.) (1985). Relapse prevention. New York: Guilford Press.

Martínez-González, J.M., Albein-Urios, N., Lozano, O. y Verdejo-García, A. (En prensa). Aspectos diferenciales del riesgo de abandono al inicio del tratamiento de la adicción a la cocaína en pacientes con trastornos de la personalidad. Adicciones.

Martínez-González J.M., Graña J.L. y Trujillo, H.M. (2011). Estudio longitudinal sobre calidad de vida, craving y ajuste psicológico en pacientes dependientes del alcohol: variaciones en función de los trastornos de la personalidad. Adicciones, 23, 227-235.

Martínez-González, J. M. y Trujillo, H. M. (2003). Tratamiento del drogodependiente con trastornos de la personalidad. Madrid: Biblioteca Nueva.

Martínez-González, J.M. y Verdejo, A. (2012). Evolución de las creencias nucleares relacionadas con la adicción en drogodependientes con y sin trastornos de personalidad. Adicciones, 24, 229-238.

Monras, M., Mondon, S. y Jon, J. (2010). Estrategias de afrontamiento en enfermos alcohólicos. Diferencias según el consumo de benzodiacepinas, trastornos de la personalidad y deterioro cognitivo. Adicciones, 22, 191-198.
Pedrero, E., Pérez, M, De Ena, S. y Garrido, C. (2005). Validación del cuestionario de Variables de Interacción Psicosocial (VIP): hacia un modelo de tratamiento de las conductas adictivas "guiado por la personalidad". Trastornos Adictivos, 7, 166-86.

Rueda, B. y Pérez-García, A. (2004). Personalidad y percepción de autoeficacia: Influencia sobre el bienestar y el afrontamiento de los problemas de salud. Revista de Psicopatología y Psicología Clínica, 9, 205-219.

Sánchez-Hervas, E., Gradoli, V.T. y Morales, E. (2004). Un modelo de tratamiento psicoterapéutico en adicciones. Trastornos Adictivos, 6, 159-66.

Sánchez-Hervas, E. y Llorente, J.M. (2012).Recaídas en la adicción a cocaína: una revisión. Adicciones, 24 (3), 269-280.

Secades, R., García-Rodríguez, O., Fernández-Hermida, J. y Carballo, J.L. (2007). Fundamentos psicológicos del tratamiento de las drogodependencias. Papeles del Psicólogo, 28, 29-40.

Sirvent, C. (2010). Predicción del abandono (vs. éxito) terapéutico en adicciones. Presentación del cuestionario VPA-30 de variables de abandono y adherencia al tratamiento en adicciones. Interpsiquis: congreso virtual de psiquiatría. $11^{\circ}$ Congreso.

Solomon, K. y Annis, H.M. (1990). Outcome and efficacy expectancy in the prediction of post-treatment drinkg behavior. British Journal of Addiction, 85, 659-665.

Urbanoski, K., Kelly, J., Hoeppner, B. y Slayma, K. (2012). The role of the terapeutic alliance in substance use disorder treatment for young adults. Journal of Substance Abuse Treatment, 43, 344-351.

Van Beek, N, y Verheul, R (2008). Motivation for treatment in patients with personality disorders. Journal of Personality Disorders, 22, 89-100.

Walton, M.A-, Blow, F.C., Bingham, C.R. y Chermack, S.T. (2003). Individual and social/environmental predictors of alcohol and drug use 2 years following substance abuse treatment. Addictive Behavior, 28, 627-42. 